\title{
When Your N >1: Drawing Meaningful Conclusions from Registry Studies
}

\author{
Mark B. Mycyk ${ }^{1}$
}

Received: 1 August 2016 / Accepted: 1 August 2016/Published online: 10 August 2016

(C) American College of Medical Toxicology 2016

Keywords Poisoning $\cdot$ Registry $\cdot$ Outcomes

Foxy methoxy. 2C-I. Lisdexamfetamine. In 12 years of publishing, many sentinel toxicologic issues were first reported here in the pages of $J M T$ in the form of case reports. It is unethical to poison subjects in a randomized prospective way, so toxicologists have traditionally relied on the details in well-written case reports about experimentation with NEW drugs or unexpected complications from new medications to identify emerging trends or propose improved treatment in future cases $[1,2]$.

But what can be interpreted or generalized from a single bedside encounter is significantly limited compared to the potential of large $\mathrm{N}$ registry studies that have become popular among researchers in recent years. On the surface, the benefits of registry studies are obvious: the $\mathrm{N}$ is $>1$ and data are collected in an organized systematic fashion. For many years, the National Poison Data System (NPDS) from our poison center network provided the best and largest registry with over two million cases each year [3]. The NPDS registry has evolved and improved significantly since its inception, and many excellent studies using NPDS data have been published, but the voluntary telephone submission of data by the public or nontoxicologist healthcare providers suffers from limitations recognizable to most of our readers [4]. In this September issue, we are pleased to feature two different registry studies that add new knowledge to what we have from NPDS and our understanding of poisoning in the USA [5, 6]. The approach and methodology of the two studies here are different, but the bottom line mes-

Mark B. Mycyk

mmycyk@cookcountyhhs.org

1 Cook County Health and Hospitals System, 1900 West Polk, 10th Floor, Chicago, IL 60611, USA sage in both is the same: poisonings continue to require specialized care and significantly increase resource utilization.

The first registry featured in this issue is the 6th Annual ToxIC report [5]. The Toxicology Investigators Consortium (ToxIC) registry was established in 2010 in an effort to fill in the missing details in NPDS by providing bedside level data on a large scale [7]. ToxIC has also evolved and improved quickly, with representation now from over 100 separate healthcare facilities where cases are eligible for the registry only if seen at the bedside by a medical toxicologist. Unlike NPDS where data are volunteered by callers with no specialized toxicology training or a formal mechanism for verifying the details, the ToxIC registry provides more credible and reliable data to analyze poisoning trends and generate new research questions. It is reassuring to see that clinically important national trends, such as the increase in severe exposure visits from sedatives last year, is a consistent finding in both NPDS and ToxIC [4, 5]. To be fair, it is important to recognize the data in ToxIC has a sampling bias, because only centers with an onsite toxicologist (or toxicology fellowship program) consulted for a case can enter data into the registry, thereby skewing the cases to an arguably sicker population. However, the distribution of active sites is sufficiently diverse, ongoing quality review has improved many data fields to mandatory responses, and several focused high level ToxIC sub-registries have been created in the last few years. As noted by the authors, "While there may be variation in agent frequencies from year to year, and new trends may emerge, overall the most common exposures, toxidromes, clinical abnormalities, and antidotes as recorded in the Registry, represent the routine practice of the specialty of Medical Toxicology. The Registry forms a representation of our specialty and the expertise that a Medical Toxicologist brings to the bedside." [5] ToxIC's 6th annual report is even better than ever and we look forward to future reports from the consortium. 
The National Hospital Ambulatory Medical Care Survey (NHAMCS) is the other large registry represented in this issue by a study done by Maryann Mazer-Amirshahi and co-authors [6]. They analyzed over eight million US ED poisoning visits over an 8-year period and found what many have front line caregivers have long suspected: poisoning-related ED visits significantly increased, were increasingly resource-intensive, and required increasingly longer lengths of ED stay or hospital admission. As an example, CT utilization for evaluating poisoned patients increased almost threefold during the course of the study.

It is important for readers to know NHAMCS is an annual survey conducted by the National Center for Health Statistics (NCHS) at the Centers for Disease Control and Prevention. To date, more studies on ED patients have published from NHAMCS than from any other US-based registry. An excellent commentary a few years ago explained why: "The popularity of NHAMCS with researchers is likely due to the rigor of the sampling methods - which permit reconstitution of a population that resembles the entire US population - and its availability online at no cost." [8] Readers of this study and other NHAMCS studies need to understand that NHAMCS does not provide an exact count of ED visits, but instead uses rigorous multistage probabilistic sampling of hospital-based visits to estimate ED utilization nationally. Unlike data entered into the ToxIC registry, NHAMCS data come from medical records abstracted by local hospital staff or Census Bureau field representatives without clinical experience. Moreover, NHAMCS changes occur yearly, including yearly changes in the hospitals sampled, changes in what variables are abstracted, and changes in how they are abstracted. As noted in a recent editorial in these pages, NHAMCS provides important policy-level data but does not provide the clinical "granularity" obtained in a well-written case report or in ToxIC, and thus the reliability of some of the data have been questioned [9]. For instance, why so many more CTs are ordered in cases of poisoning cannot be answered from NHAMCS. But it is important to recognize here that Mazer-Amirshahi and her coauthors have bedside level ED and toxicology experience, and that shows in how they handled the data, acknowledged their limitations, and proposed future directions. Unlike some other NHAMCS registry studies done by researchers with no bedside experience, this is a study by authors who actually practice frontline medicine. It is also important to remember that NHAMCS includes non-academic centers, therefore providing a better idea of national trends than some other published registries. Poisonings that result in ED evaluation, regardless of the severity, are indeed resource-intensive visits today. The findings by Mazer-Amirshahi provide additional perspective to what we have from NPDS and ToxIC.

The ideal database would be one that accurately records relevant clinical information for every relevant patient, but we currently do not have that in place in the USA. The national effort to make all visits recorded in an easily accessible electronic health record may result in that type of registry in the future. Until then, understanding current poisoning trends and generating new study leads demands critical and careful evaluation of the many different registries available right now. The findings in a registry are only as good as the origin of the raw data and the experience of the authors using it. Just because the $\mathrm{N}$ of any registry is greater than the $\mathrm{N}=1$ in a case report does not make the registry better. We need to remember none of these registry studies are perfect - the data are not infallible and sometimes the conclusions are not necessarily more reliable than in a well-documented case report. Each registry study offers meaningful data to readers who know how to use them. Read the current ToxIC report. Read the NHAMCS study here. And read the annual NPDS. Develop your own conclusions and provide feedback to the stewards of these registries so they can make them better. And finally, look forward to the day in the distant future when we have a single collaborative registry database with all the relevant information needed for clinicians, scholars, and policy makers to improve outcomes in poisoned patients.

\section{Compliance with Ethical Standards}

Conflicts of Interest None

Disclosures None

Sources of Funding None

\section{References}

1. Ruha A-M. The case report: a tool for the toxicologist. J Med Tox. 2009;5:1-2.

2. Mycyk MB. Emerging drugs of abuse: what was new yesterday is new today. J Med Tox. 2012;8:1-2.

3. Mowry JB, Spyker DA, Brooks DE, McMillan N, Schauben JL. 2014 annual report of the American Association of Poison Control Centers' National Poison Data System (NPDS): 32nd annual report. Clin Toxicol (Phila). 2015;53(10):962-1147.

4. Hoffman RS. Understanding the limitations of retrospective analyses of poison center data. Clin Toxicol (Phila). 2007;45(8):943-5.

5. Farrugia LA, Rhyee SH, Campleman SL, Ruha A-M, Wiegand T, Wax PM, Brent J. The Toxicology Investigators Consortium Case Registry - the 2015 Experience. J Med Toxicol. 2016;12(3).

6. Mazer-Amirshahi M, Sun C, Mullins P, Perrone J, Nelson L, Pines JM. Trends in emergency department resource utilization for poisoning-related visits, 2003-2011. J Med Toxicol. 2016;12(3).

7. Wax PM, Kleinschmidt KC, Brent J. ACMT ToxIC Case Registry Investigators. the Toxicology Investigators Consortium (ToxIC) Registry. J Med Toxicol. 2011;7(4):259-65.

8. Cooper RJ. NHAMCS: does it hold up to scrutiny? Ann Emerg Med. 2012;60(6):722-5.

9. Connors NJ, Nelson LS. The devil is in the details but the details are not in NHAMCS. J Med Toxicol. 2016;12(2):145-7. 